\title{
Local Cooperative Relay for Opportunistic Data Forwarding in Mobile Ad-hoc Networks
}

\author{
Zehua Wang, Cheng Li, and Yuanzhu Chen \\ Memorial University of Newfoundland \\ St. John's, NL, Canada \\ \{zehua.wang, licheng, yzchen\}@mun.ca
}

\begin{abstract}
-opportunistic data forwarding draws more and more attention in the research community of wireless network after the initial work ExOR was published. However, as far as we know, all existing opportunistic data forwarding only use the nodes which are included in the forwarder list in the entire forwarding progress. In fact, even if a node is not a listed forwarder in the forwarder list, but it is on the direction from source node to destination node, and when it successfully overhears some packets by opportunity, the node actually can be utilized in the opportunistic data forwarding progress. In this paper, we propose the local cooperative relay for opportunistic data forwarding in mobile ad-hoc networks. In general, three contributions we have in this paper, 1) we open more node to participate in the opportunistic data forwarding even though the nodes are not included in the forwarder list, 2) we propose the procedure to select the best local relay node, namely the helper-node, from many candidates but require no inner communication between them, 3) the helper-node is selected just when it is needed, and the such real time selection can tolerate and bridge vulnerable links in mobile networks.
\end{abstract}

Index Terms-Opportunistic data forwarding, local relay, local retransmission, coordination, scoring function.

\section{INTRODUCTION}

A multi-hop wireless network is a communication network, where nodes which can not direct communicate with each other will require other nodes to forward data. It can operate without existing infrastructure and supports mobile users. It was initially proposed to meet the requirements of battlefield communications, and after decades' development, it is also used in civilian applications to support a community network. A great deal of research have been published since 1980's [1] and the network layer has received the most attention in the research community. As the result, a great deal of routing protocols have been proposed [2].

Despite the amount of efforts have been made in routing protocol design for multi-hop wireless network, the data forwarding, on the other hand, follows pretty much the same as that in IP forwarding in the Internet. IP forwarding was designed for multi-hop wired networks, where one transmission can only be detected by the node on the other side of the same cable. However, in wireless networks, when a packet is sent

This work was supported in part by the Natural Sciences and Engineering Research Council (NSERC) of Canada (Discovery Grants 293264-07 and 327667-2010, and Strategic Project Grant STPGP 397491-10). out over a wireless channel, it may be detected by all nodes within its transmission range. Within the long period of the research history, overhearing a packet by unintended receivers is considered negative completely, i.e., interference. Thus, the purpose of wireless networking was to make wireless networks as good as the wired ones. However, this ignores the broadcast nature of wireless communication links. If we further consider the mobile ad hoc networks, we have to tame and utilize its broadcasting nature rather than fighting it. Cooperative communication is an effective approach to achieve our goal.

However, little research had been done to develop cooperative communication at the link layer and above, until ExOR [3]. ExOR is a milestone in this area and it utilizes the broadcast nature of wireless links to achieve cooperative communication at the link and network layers in wireless mesh networks. We will review ExOR in Section II but in general, ExOR has two important contributions. First, all nodes on the route are contained in each data packet as a forwarder list, so any node in the forwarder list can forward the received packets. Second, the further node away from source in the route has higher priority to forward the received packets, so the long-haul transmission can be utilized, and upstream nodes avoid duplicate transmissions by overhearing the packets transmitted by downstream nodes.

From the evolution concept of the forwarding techniques in wireless networks, ExOR does utilize the overhearing in wireless networks by including all nodes on the route to be intended next forwarder, but it still limits us to further explore the broadcast nature. That is because only the nodes included in the forwarder list can participate in the opportunistic data forwarding and benefit from the broadcast nature to enhance the network performance. In the paper, we extend the idea in ExOR and propose the local cooperative relay for opportunistic data forwarding. Contributions in our solution are highlighted as follows.

- Not only the nodes contained in the forwarder list, but also other nodes that are on the direction from source to destination will be used in the opportunistic data forwarding progress.

- We proposed a procedure with a new scoring function to select a helper-node from many candidates but require no 
inner communication between them, and the selected one participate in the opportunistic data forwarding directly.

- The helper-node is selected in real time. It is suitable to support mobile network and fix the broken links caused by nodes' mobility.

The rest of this article is organized as follows. In Section II, we will review the related work and introduce our motivation. Section III will present the details in the proposed local cooperative relay scheme. The scoring function used in our implementation will be introduced in Section IV. The performance evaluations of the proposed scheme are presented in Section V. Section VI concludes the article with outlook to future research.

\section{RELATED WORK AND MOTIVATION}

In this section, we will first provide a review of ExOR and summarize its derivations, and then we will highlight the motivation of our work.

\section{A. ExOR and the derivations}

ExOR [3] is an explorative cross-layer opportunistic data forwarding technique in multi-hop wireless networks by Biswas and Morris. It fuses the MAC (Medium Access Control) and network layers so that the MAC layer can determine the actual next-hop forwarder after transmission depending on the transient channel conditions at all eligible downstream nodes. Nodes are enabled to overhear all packets transmitted in the channel, whether intended for it or not. A multitude of forwarders can potentially forward a packet as long as it is included on the forwarder list carried by the packet. In fact, the forwarder list in ExOR contains all nodes on the entire route from source to the destination, and a node which is closer to the destination has the higher priority to forward the packet it just received. Thus, if a packet is heard by a listed forwarder closer to the destination with a good reception condition, this long-haul transmission should be utilized. Otherwise, shorter and thus more robust transmissions can always be used to guarantee reliable progress.

The idea of ExOR inspired a number of interesting extensions. MORE [4] enhances ExOR to further increase the spatial reuse in a single flow from source to destination via intra-flow network coding [5]. Leontiadis and Mascolo [6] and Yang et al. [7] propose using position information for the routing module to support mobile multi-hop wireless networks. Therein, it is assumed that every node is aware of the positions of all other nodes in the network.

\section{B. Motivation of our work}

ExOR is an evolution on the forwarding techniques of wireless networks. In particular, before ExOR, the IP forwarding only has one intended next hop which is specified by the next hop MAC address. Hence, the unicast in wireless transmission is a tricky concept where the MAC address is used as a filter to compel the wireless nodes to forget their broadcast nature. ExOR adds the forwarder list in every data packet, and all nodes in the forwarder list could be next hop by opportunity.
However, from another point of view, ExOR also has the limitation that only the nodes included on the forwarder list can benefit from broadcast nature. In fact we can explore the broadcast nature and receiver diversity in a deeper level. Let us take Figure 1 as an example. We assume node $A$ is the

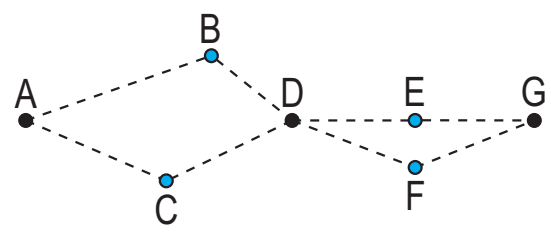

Fig. 1. Topology example for motivation

source node and node $G$ is the destination, and the forwarder list in ExOR contains nodes $A, D$, and $G$. We further assume $B$ and $C$ are two mutual neighbors of two adjacent listed forwarders $A$ and $D$, and $E$ and $F$ are two mutual neighbors of two adjacent listed forwarders $D$ and $G$. According to the operation in ExOR, the forwarder list is contained by every packet and all the packets are transmitted by broadcast, so by overhearing the forwarder list $A, D$, and $G$, both $B$ and $C$ can be notified that they are on the direction from source to destination. With the same reason, $E$ and $F$ know that they are on the direction from source to destination as well. Hence, if nodes $B, C, E$, and $F$ overhear some data packets that the downstream nodes fail to receive, they may give a hand to forward them. That makes us to think about how to utilize the opportunistic data forwarding one step further than ExOR.

As far as we know, this paper is the first work to try to open more nodes which are not included in the forwarder list to participate in opportunistic data forwarding.

\section{Design - Challenges AND Solutions}

In this section we will present the details in our design. In the motivation from Section II-B, we have generally talked about the basic idea, but some challenges are still need to be solved.

\section{A. Challenges}

We do want to use the mutual neighbors of two adjacent listed forwarders to be the helper-nodes, however, this condition is not sufficient. To illustrate this, we assume we have a topology like Figure 2, where we assume $X$ and $Y$ are two

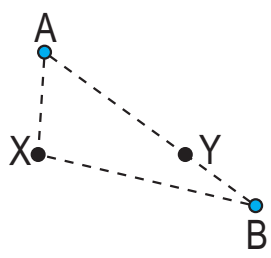

Fig. 2. Example of unsuitable nodes

adjacent listed forwarders, and $A, B$ are the mutual neighbors of them. In this situation, neither $A$ nor $B$ is suitable to be the helper-node because the distance from the listed forwarder to either $A$ or $B$ is longer than the distance between listed 
forwarders directly. Hence the first challenges in our design is how to validate the suitability of a mutual neighbor between two adjacent listed forwarders, and the helper-nodes can only be selected from valid local relay candidates.

As the topology in Figure 1 indicates, there may be more than one mutual neighbors between two adjacent listed forwarders, and also we may have more than one valid candidates. When we want the valid local relay candidates to give a hand on the opportunistic data forwarding, we do not want duplicated transmissions or excessively contentions between them. Because these valid candidates are not included in the forwarder list, how to coordinate them is the second challenge in our design.

To coordinate multiple nodes in wireless networks, the extra communications are usually required, which introduces more overhead and longer delay. Here we want to design a procedure that introduces no delay and minimum overhead, and this becomes our third challenge.

\section{B. Solutions}

In this paper, we use Received Signal Strength Indicator (RSSI) to evaluate the quality of links. According to the work done by Charles Reis et al. [8] and Mei-Hsuan Lu et al. [9], RSSI can be reported by almost all commodity wireless cards and a greater RSSI usually indicates a better wireless channel quality. Furthermore, in free space the ideal received signal strength is inversely related with the $n^{\text {th }}$ power of the distance between the transmitter and receiver. If we denote the RSSI received between a pair of nodes $M$ and $N$ is $R(M, N)$ and use $d_{M, N}$ to present the distance between them, in ideal free space we have

$$
R(M, N)=C_{1} \times d_{M, N}^{-n},
$$

where $C_{1}$ is a constant, and the free space assumption also indicates us that all links are symmetric, e.g., $R(M, N)=$ $R(N, M)$.

According to Formula 1, the receiver side can estimate the distance of the transmitter by the RSSI detected. Hence we propose that node $i$ is a valid local relay candidate if and only if it satisfies following two conditions,

- $i$ is the neighbor of both $X$ and $Y$ which are two adjacent listed forwarders.

- $i$ has better RSSI to both $X$ and $Y$ than the RSSIs from $X$ to $Y$ or $Y$ to $X$ directly, i.e.,

$$
\left\{\begin{array}{l}
R(i, X) \geq R(Y, X) \\
R(i, Y) \geq R(X, Y) .
\end{array}\right.
$$

To coordinate the valid local relay candidates we propose to select the best one only, namely the helper-node. This decision makes sense because the Formula $2 \mathrm{a}$ and $2 \mathrm{~b}$ together constrain a small region for valid local relay candidates between two adjacent listed forwarders, as the result, even if their are many candidates exist in the same region, their channel conditions are early the same.

Hence, the next question is how to make all valid local relay candidates make an identical decision on which one is the best. This question would be solved based on the RSSIs as well. However, in our design, every node is required to maintain the RSSIs for all links within its two hops neighbors. This requirement can be easily met by making every node broadcast its one hop neighbors' link qualities with its beacon periodically. Now, let us take the topology in Figure 3 as an example. Both $A$ and $B$ are two neighbors nodes of $X$ and $Y$

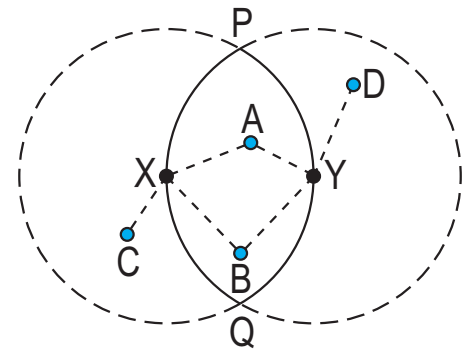

Fig. 3. Topology example for design

which are assumed as the listed forwarders. $A$ and $B$ are in the oliver region surrounded by arc $\widehat{P X Q}$ and $\widehat{P Y Q}$, so they are valid local relay candidates according to Formula $2 \mathrm{a}$ and $2 \mathrm{~b}$. Note node are required to maintain the RSSI of each link within two hops and they broadcast their one hop neighbors' link qualities periodically, so node $A$ (or $B$ ) knows it is not the unique valid relay node candidate and it has a competitor $B$ (or $A$ ). With the above reason as well, node $A$ (or $B$ ) not only knows the RSSIs between itself to $X$ and $Y$ but also has the RSSIs between its competitors to both listed forwarders. As a result, no matter how many valid local relay candidates exist in the oliver region, the RSSIs between these valid local relay candidates to the listed forwarders are all shared with others. Note we use RSSIs reported by $X$ and $Y$ to estimate the link qualities, so the estimation are performed with same set of RSSIs by all valid local relay candidates. Therefore, every node will make an identical decision that if it is the helper-node or not, and with no gossip. How to design a scoring function with the set of RSSIs as the input is an independent topic, and we will introduce our scoring function in Section IV.

To make the selected helper-node participate in the forwarding process without collisions with other listed forwarders, we let the helper-node wait a period of time which is longer than the downstream listed forwarder but shorter than the upstream one. This is feasible because the helper-node can find out its priority according to its neighbors' priorities in the overheard forwarder list. As well, the best local relay node can calibrate its forwarding time by overhearing its downstream node's transmission, and it only forwards the overheard packets that have never been forwarded by any of its downstream nodes.

\section{SCORING FUNCTION}

In previous section we have introduced that all valid local relay candidates share the same sets of RSSIs reported by listed forwarders. The scoring function helps every valid local relay candidate find out if it is the helper-node. Intuitively, 
a node has a better position than any others if it is on the middle point between two listed forwarders, and if a node is too close to either one of the two listed forwarders, or it is far away from both of them, it would not be a good choice to be the helper-node. In fact, the scoring function is not unique in our studying, and any function meets the above intuition may make sense.

Within the scoring function we proposed, the helper-node must have a smaller score than its competitors. In particular, for each relay candidate $i$ between two adjacent listed forwarders $X$ and $Y$, the scoring function is given by

$$
\begin{aligned}
& s(i)=\left(\sqrt[4]{\frac{1}{R(i, Y)}}+\sqrt[4]{\frac{1}{R(i, X)}}\right) \times \\
& \left(\ln \sqrt[4]{\frac{\max \left(\frac{1}{R(i, Y)}, \frac{1}{R(i, X)}\right)}{\min \left(\frac{1}{R(i, Y)}, \frac{1}{R(i, X)}\right)}}+\ln \left(\sqrt[4]{\frac{1}{R(i, Y)}}+\sqrt[4]{\frac{1}{R(i, X)}}\right)\right) .
\end{aligned}
$$

To prove the scoring function is make sense, we replace the $R$ function in Formula 3 by the distance according to Formula 1, and after the simplification we have

$$
\begin{array}{r}
s(i)=\frac{\left(d_{i, Y}^{\frac{n}{4}}+d_{i, X}^{\frac{n}{4}}\right)}{4 \times \sqrt[4]{C_{1}}} \times \\
\left(n \times \ln \frac{\max \left(d_{i, Y}, d_{i, X}\right)}{\min \left(d_{i, Y}, d_{i, X}\right)}+4 \times \ln \left(d_{i, Y}^{\frac{n}{4}}+d_{i, X}^{\frac{n}{4}}\right)-\ln C_{2}\right) .
\end{array}
$$

where $C_{1}$ and $C_{2}$ are constants.

Note $n$ in Formula 4 is the pass loss parameter, and usually it takes value 2,3 , or 4 . We plot the contours of the proposed scoring function with different values of $n$, and they have similar shape and properties in Matlab. Because of the limit of space, we only present the result at the condition $n=$ 2 in Figure 4 and Figure 5. The top view shows us the

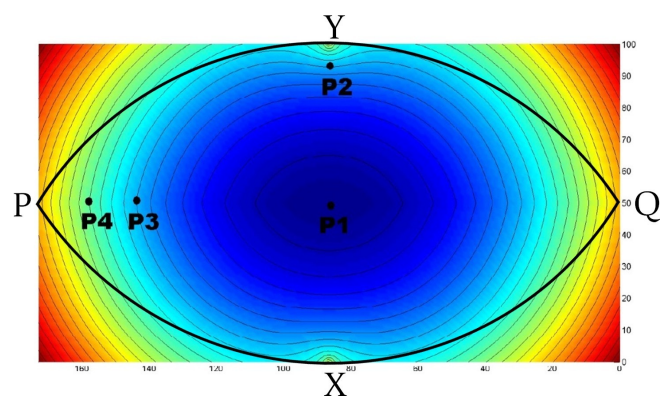

Fig. 4. Contour of scoring function : top view

circumscribed quadrilateral of the olivary shape in Figure 3, and to save the space, we rotated the plotted figure 90 degree anticlockwise. Furthermore, the profile in Figure 5 intuitively shows us the shape of the scoring function in 3D. We can clearly see that the scoring function gives the minimum value at the middle point between two listed forwarders, such as $p_{1}$ in the figures. If a relay node is quite close to either one of the listed forwarders or it is far from both of them, the node

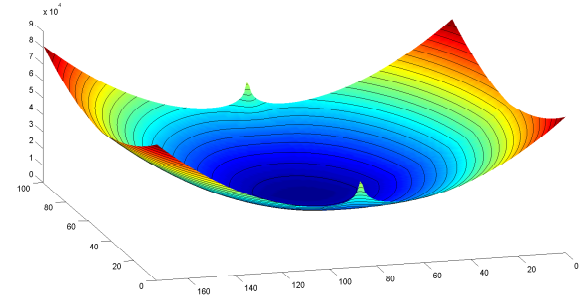

Fig. 5. Contour of scoring function : side view

has less chance to be taken as the helper-node, such as $P_{2}$ and $P_{4}$. Furthermore, we have $P_{2}$, which has the same score with $P_{3}$ but smaller score than $P_{4}$ according to our scoring function, and that is reasonable because $P_{4}$ is far away from both of listed forwarders while $P_{2}$ is close to one of them.

The $4^{\text {th }}$ root calculation in Formula 3 can also be changed to other $n^{\text {th }}$ root calculation, and they actually have the similar surfaces as we plotted in Figure 4 and Figure 5.

\section{Performance eValuation}

We study the performance of local cooperative relay in opportunistic data forwarding by running computer simulation with Network Simulation ns-2 (version 2.34). We compare two different kinds of opportunistic data forwarding schemes in mobile networks, one is under the condition that the local cooperative relay module is enabled, and the other is with the local cooperative relay module disabled. The routing module we selected is Proactive Source Routing (PSR) [10] and we test UDP data flow in our network. Generally speaking, we find the opportunistic data forwarding scheme with the local cooperative relay module enabled outperforms the one with the local cooperative relay module disabled.

\section{A. Experiment settings}

In our work, we choose the Nakagami propagation model to test the effectiveness of the proposed scheme. In ns-2, when a node has received a packet, it first calculates the received power using path loss based on the Frii Free-Space model. This value is compensated with Nakagami's fluctuation before further processing. We configure the nominal data rate at the 802.11 links to $1 \mathrm{Mbps}$,

In modeling node motion, we adopt the random waypoint model to generate the simulation scenarios. In this model, each node moves towards a series of target positions. The rate of velocity for each move is uniformly selected from $\left[0, v_{\max }\right]$. Once it has reached a target position, it is moving towards the next position directly.

We inject CBR (constant bit rate) data flows in the network, which are carried by UDP. Specifically, a source node generates 50 packets every second, each has a payload of 1000 bytes. This translates to a traffic rate of $400 \mathrm{kbps}$ injected by a node. When comparing different opportunistic data forwarding schemes with the local cooperative relay enabled or not, we record the packet delivery ratio (PDR), i.e. the fraction of packets received by the destination out of all the packets injected, and end-to-end delay average. We 
observe that the local cooperative relay can help to enhance the PDR and reduce the end-to-end delay.

\section{B. Performance versus network dimension}

We first make performance comparison between two opportunistic data forwarding schemes that with the local cooperative relay enabled and disabled. In particular, we have network tomographies of $l \times l\left(\mathrm{~m}^{2}\right)$, where $l=450,500,550, \ldots, 950$. We deploy 50 nodes in each of these network dimensions to test the protocols with differing node densities, and every node moves randomly with waypoint model at $v_{\max }=20$ $\mathrm{m} / \mathrm{s}$. For each dimension scenario, we test performances of such two schemes in transporting CBR data flows between a randomly selected source-destination pair. We repeat this process 20 times for a given scenario. We measure the PDR, end-to-end delay for both protocols and average them over the 20 repetitions of each scenario, as plotted in Figures 6 and 7.

We observe that when the network dimension is relatively small, opportunistic data forwarding with local cooperative relay disabled has better PDR. In particular, when the side length of the square network boundary grows from $450 \mathrm{~m}$ to $500 \mathrm{~m}$, the opportunistic data forwarding with local cooperative relay disabled outperform that with proposed scheme enabled. When the side length of the square boundary keep on increasing from $500 \mathrm{~m}$, the opportunistic data forwarding with local cooperative relay module enabled outperform its opponent, and the greater the side length, the more obvious PDR gain could be achieved.

To investigate the reason behind such a phenomena, we plot the profit and loss of the proposed scheme in Figure 8. The left scale of Figure 8 records the number of packets forwarded by helper-nodes and the number of collisions happened on listed forwarders caused by helper-nodes. The right scale gives us the ratio of such two numbers. It is obvious that both the forwarding times on helper-nodes and the collisions they caused increase when the network dimension goes up, however, the forwarding times increase faster than the collisions they caused. In our further investigation, we find the forwarding times on helper-nodes increase because each route has more hops from source to destination with the increasing network dimensions. In contrast, we find the number of collisions does not not goes up directly with the growth of hops in routes, but with a slower rate. That is because, to avoid collision, every node has been configured to wait a different amount of time according to its priority, but longer route reduces the number of packets in each fragment [3]. As the result, the upstream node more likely fails to detect the downstream node's transmission, so nodes can not perfectly calibrate the time to start their forwarding, which actually is the real reason causes the collisions. Hence, the time of collisions grows slower than the time of forwarding contributed by our helper-nodes. Meanwhile, because there are less hops on each route in small dimension networks and considering node will retry several rounds before dropping packets in opportunistic data forwarding, the contribution of local cooperative relay in dense network is limited. When the network dimensions increases, our proposed scheme shows its advantage.

From Figure 7 we can see in dense network, the packet end-to-end delay in both tested schemes are nearly the same. However, in sparse network, the opportunistic data forwarding with our proposed scheme has shorter packet end-to-end delay. That is because the local cooperative relay will rescue many packets to avoid them to be delivered from original source again.

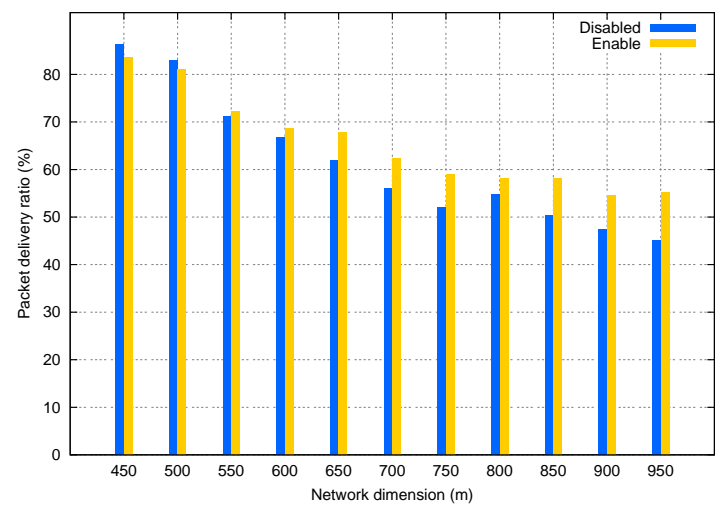

Fig. 6. PDR vs. network dimension

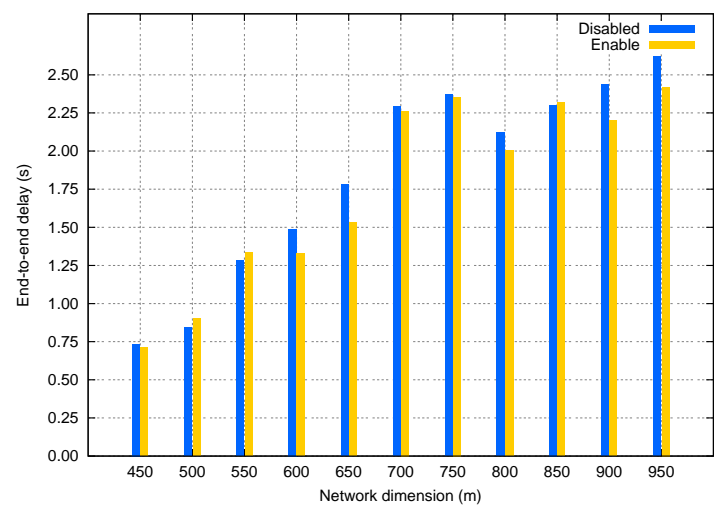

Fig. 7. Packet delay vs. network dimension

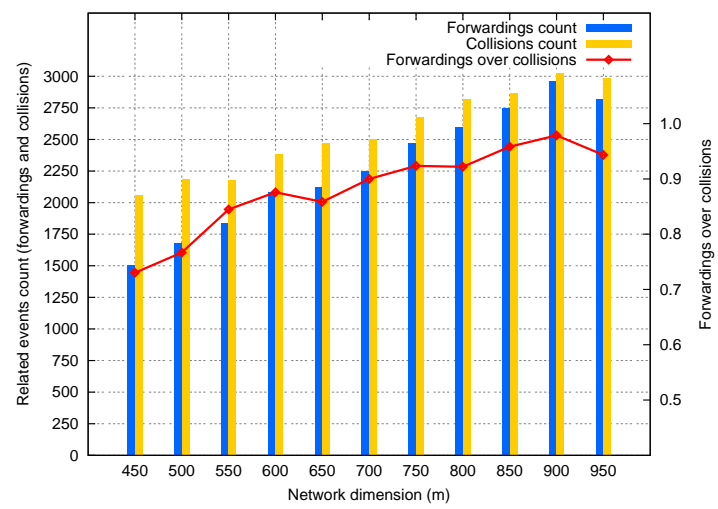

Fig. 8. Reasons analysis of PDR reversion 


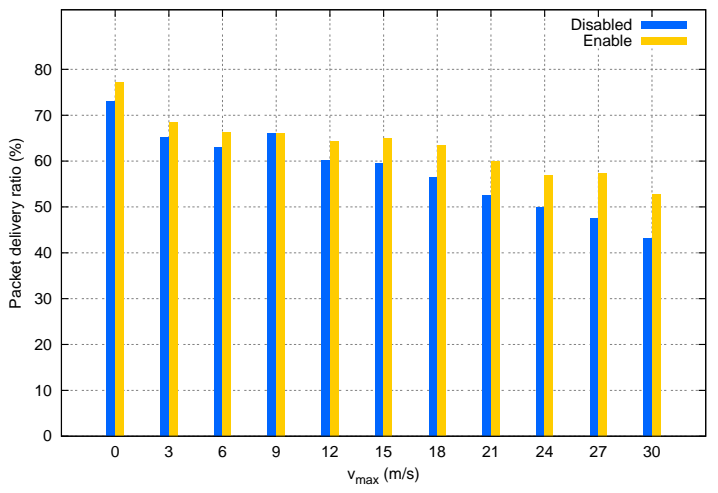

Fig. 9. PDR vs. node velocity

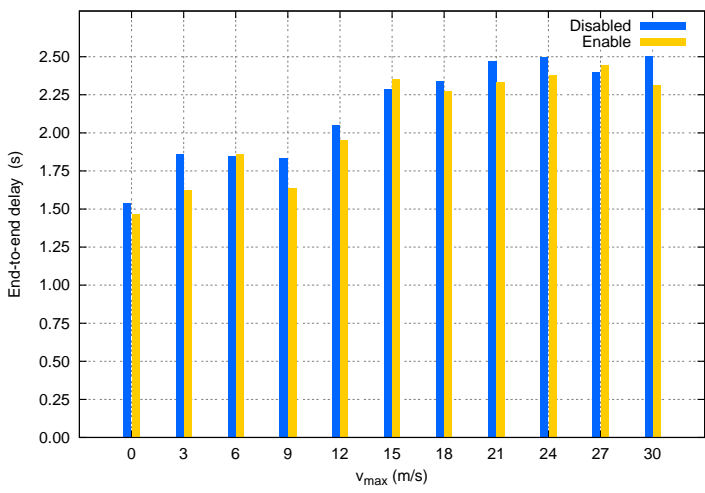

Fig. 10. Packet delay vs. node velocity

\section{Performance versus velocity}

We conduct another set of tests in a network of 50 nodes deployed in a $700 \times 700\left(\mathrm{~m}^{2}\right)$ space with a varying $v_{\max }$, where $v_{\max }=0,3,6, \ldots, 30(\mathrm{~m} / \mathrm{s})$. For each velocity scenario, we test two different opportunistic data forwarding progresses - with or without the local cooperative relay scheme. All tests are between a randomly selected source-destination pair as well, and we repeat this process 20 times for a given scenario and collect the average PDR and end-to-end delay.

From Figure 9, we find for all velocity scenario, the opportunistic data forwarding with local cooperative relay outperforms the other for the same reasons as we analyzed in Section V-B. What makes us feel interesting is that even thought the PDRs in both tests decrease together when nodes move faster, the PDR gain of local cooperative relay increases when the velocity goes up. That is due to the helper-node is selected in real time and it can effectively bridge the broken links caused by the mobility. Therefore, the local cooperative relay provides more effectiveness to fix up the broken links when the node velocity increases.

Also, the local cooperative relay provides shorter end-toend delay because of the similar reason we analyzed before.

\section{CONCLUDING REMARKS}

In this paper we proposed the local cooperative relay in opportunistic data forwarding. The local cooperative relay extend the opportunistic data forwarding one step further than ExOR by using more nodes which are not contained in the forwarder list to give a hand in opportunistic data forwarding. Furthermore, the transient high quality links may quite easily break in mobile networks, but the local cooperative relay we proposed in this paper can effectively bridge the broken links and maintain a robust topology. We also proposed a scoring function based on the RSSI, which can effectively select the best node to be the helper-node without additional overhead.

The local cooperative relay we proposed here is just the first step to remove the constraints given by ExOR and enable more nodes to participate in the opportunistic data forwarding. More nodes that involved in the opportunistic data forwarding can further explore the broadcast nature and receiver diversity. However, involving more nodes in opportunistic data forwarding makes the forwarder list no longer sufficient to regulate the sequence of data transmissions, so how to reduce the coordination overhead and depress the collisions have become promising topics in the further research.

\section{REFERENCES}

[1] I. Chlamtac, M. Conti, and J.-N. Liu, "Mobile Ad hoc Networking: Imperatives and Challenges," Ad Hoc Networks, vol. 1, no. 1, pp. 1364, July 2003.

[2] R. Rajaraman, "Topology control and routing in ad hoc networks: A survey," SIGACT News, vol. 33, pp. 60-73, June 2002.

[3] S. Biswas and R. Morris, "ExOR: Opportunistic Multi-Hop Routing for Wireless Networks," in Proceedings of ACM Conference of the Special Interest Group on Data Communication (SIGCOMM), Philadelphia, PA, USA, August 2005, pp. 133-144.

[4] S. Chachulski, M. Jennings, S. Katti, and D. Katabi, "Trading Structure for Randomness in Wireless Opportunistic Routing," in Proceedings of ACM Conference of the Special Interest Group on Data Communication (SIGCOMM), Kyoto, Japan, August 2007, pp. 169-180.

[5] C. Fragouli, J.-Y. L. Boudec, and J. Widmer, "Network Coding: an Instant Primer," SIGCOMM Computer Communication Review, vol. 36, pp. 63-68, January 2006.

[6] I. Leontiadis and C. Mascolo, "GeOpps: Geographical Opportunistic Routing for Vehicular Networks," in Proceedings of the IEEE International Symposium on a World of Wireless Mobile and Multimedia Networks (WoWMoM), Helsinki, Finland, June 2007, pp. 1-6.

[7] S. Yang, F. Zhong, C. K. Yeo, B. S. Lee, and J. Boleng, "Position Based Opportunistic Routing for Robust Data Delivery in MANETs," in Proceedings of the 2009 IEEE Conference on Global Telecommunications (GLOBECOM), Honolulu, Hawaii, USA, December 2009, pp. $1325-1330$.

[8] C. Reis, R. Mahajan, M. Rodrig, D. Wetherall, and J. Zahorjan, "Measurement-Based Models of Delivery and Interference in Static Wireless Networks," in Proceedings of ACM SIGCOMM, August 2006, pp. 51-62.

[9] M.-H. Lu, P. Steenkiste, and T. Chen, "Design, Implementation and Evaluation of an Efficient Opportunistic Retransmission Protocol,' in Proceedings of the 15th Annual International Conference on Mobile Computing and Networking (MobiCom). New York, NY, USA: ACM, 2009, pp. 73-84.

[10] Z. Wang, Y. Chen, and C. Li, "A New Loop-Free Proactive Source Routing Scheme for Opportunistic Data Forwarding in Wireless Networks," IEEE Communications Letters, vol. 15, pp. 1184-1186, November 2011. 